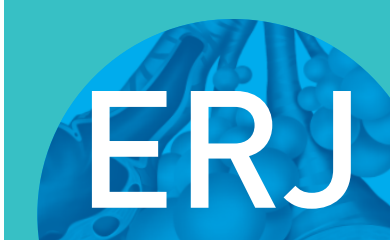

open research

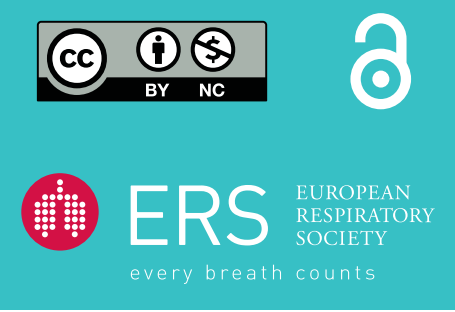

\section{Sarcoidosis: patient treatment priorities}

To the Editor:

Sarcoidosis is a rare disease with the number of patients in the European Union (28 member states), Norway, Iceland and Lichtenstein estimated as $102000[1,2]$. Some $40 \%$ of individuals experience sarcoidosis as a chronic condition, while $>60 \%$ of individuals find that the disease resolves within 2-5 years [3]. However, it has been shown that patients can still have symptoms that result in a reduced quality of life (QoL) even after clinical signs of disease have disappeared. These symptoms include fatigue, pain, everyday cognitive failure, small fibre neuropathy, exercise limitation and depressive symptoms $[4,5]$. Findings from a recent survey of patients and their partners in the Netherlands also showed that sarcoidosis has a significant impact on their lives [6].

In 2016, a European Respiratory Society (ERS) task force was set up to develop an international clinical guideline on sarcoidosis treatment to provide evidence-based recommendations for healthcare professionals. Sarcoidosis outcomes are traditionally governed by clinical measures, such as blood tests, pulmonary function tests (PFTs) and imaging, but to inform the development of this guideline recommendation, a survey was designed to gather views about which treatment outcomes matter most to sarcoidosis patients [7].

The European Lung Foundation (ELF) supported the facilitation of patient input into the task force via its Sarcoidosis Patient Advisory Group (PAG), which comprises patient organisation representatives from nine countries.

The anonymous survey asked patients to rate the following seven outcomes: QoL, functionality, PFTs, blood tests, imaging, adverse events and survival. Outcomes were rated using a five-point scale (1=extremely important, 2=very important, 3=moderately important, 4=slightly important, 5=not important at all, and $6=$ no opinion). The survey was available online in six languages (Dutch, German, English, Italian, Spanish and French) during January and February 2018. The data were analysed using a calculation of the mean across all ratings. A themed analysis of all the respondents' comments was also performed.

The limitations of the survey included that it was self-selecting. People diagnosed with sarcoidosis were invited to participate via promotion, mainly through sarcoidosis patient organisation networks. As it was only available online, access was restricted to those who were technologically aware and had access. Finally, the survey was only available in specific languages, limiting responses. Despite these limitations, one of the strengths of the survey was its high response rate, with 1842 responses received (692 Dutch, 528 German, 338 English, 148 Italian, 107 Spanish and 29 French).

Figure 1 shows the quantitative results from the survey. QoL and functionality were rated the most important outcomes, while blood tests and PFTs were least important. Imaging was ranked as a more important outcome than blood tests or PFTs.

The results were very similar across all language surveys with just a few narrow variations, suggesting that these findings are relevant to people with sarcoidosis in different countries and health systems.

The ELF Sarcoidosis PAG would like to highlight three interlinking messages based on the quantitative and qualitative data received.

$@$ ERSpublications

Sarcoidosis patient survey reveals QoL and functionality are required as core outcomes in treatment and care, along with more multidisciplinary working by clinicians and the establishment of specialist sarcoidosis centres in every European country http://ow.ly/DTvt30mQnqc

Cite this article as: Baughman RP, Barriuso $\mathrm{R}$, Beyer $\mathrm{K}$, et al. Sarcoidosis: patient treatment priorities. ERJ Open Res 2018; 4: 00141-2018 [https://doi.org/10.1183/23120541.00141-2018].

Copyright $\odot$ ERS 2018. This article is open access and distributed under the terms of the Creative Commons Attribution NonCommercial Licence 4.0. 


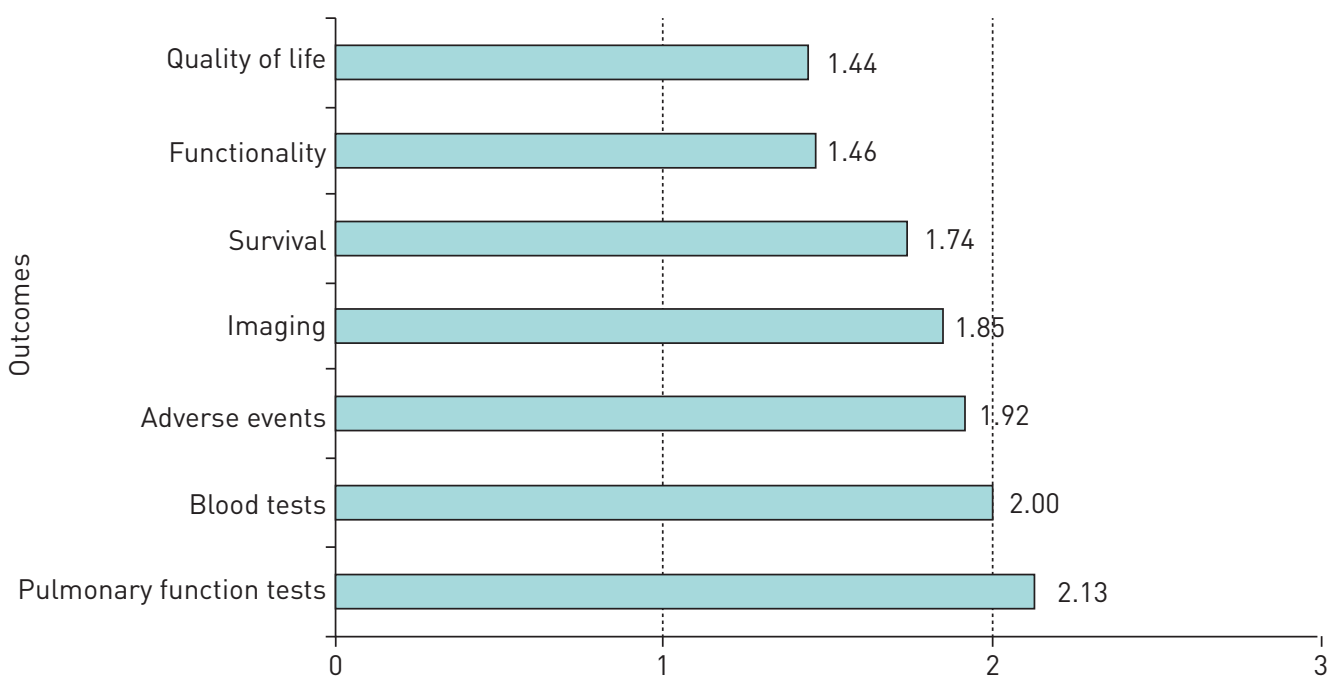

FIGURE 1 Summary of the ranking for the outcome of the seven survey questions. The mean scores were between 1 (extremely important) and 3 (moderately important).

1) QoL and functionality should be included in treatment outcomes. The survey findings strongly show that respondents want QoL and functionality to be included as outcomes in their treatment and care. In an editorial published in 2017, the view was expressed that there are only two indications for the treatment of sarcoidosis: fear of danger and significant impairment in quality of life, described as Wells Law [8]. However, in the main, clinicians focus on radiographs, blood tests, PFT and severity tools to assess and design a treatment plan. As a result, the assessment of cognitive and psychological aspects relating to QoL and functionality are less likely to be incorporated [5]. A specific comment which resonated with the group was: "Scans, tests and examinations are of course crucial in the treatment, but the well-being and the quality of life determine how much I suffer from sarcoidosis. And that is the most important thing."

Our survey also highlighted that assessing ability to work and provision of supportive measures in this area were additional important factors for consideration. The survey results reinforce the view that clinical indicators should not be the only target of treatment and that sarcoidosis clinicians should be encouraged to assess patient-reported aspects so that they can be incorporated into care.

2) Clinicians should work in a multidisciplinary way to ensure a holistic approach. In sarcoidosis, it is important to identify every symptom and treat the patient as a whole. The survey results stress that treating each patient as a person and not as a lung disease is imperative. This is particularly important for multisystem diseases to ensure that specialists can contribute their expertise at the same time to enable quicker, accurate and comprehensive diagnosis, treatment and follow-up.

Access to a range of services, such as pain management and psychological support, is important to achieve this. Psychological support was identified as a potentially powerful instrument to increase QoL and help clinicians to tailor the therapies more appropriately. Two specific comments that summarised the feeling of the group were: "I think that although mental health is included in the definition of quality of life in this survey it is important enough to have its own category. Often sarcoidosis patients suffer from depression and low mood. Going through tests, chasing appointments, taking drugs every day and seeing health deteriorate definitely takes its toll on mental health and it's important because it will ultimately underpin recovery and overall health and wellbeing." And: "A multidisciplinary approach with an emphasis on functionality is important. I have never had another doctor see me other than my internist".

3) Sarcoidosis specialists and specialist centres are needed in every country. Sarcoidosis can be difficult to diagnose and treat when there is a lack of expertise, particularly for the rarer manifestations such as neurological sarcoidosis. There were repeated requests made by respondents in our survey for an increase in the number of sarcoidosis specialists and centres. The authors support a proposal by the National Heart, Lung and Blood Institute [9] for centres of excellence to be established. One specific comment which was felt to reflect this was: "It is important to increase the number of centres that deal with this disease as seen for other more widespread but also incurable diseases."

To conclude, we hope that these survey findings will reinforce the value in investing in patient-centred sarcoidosis healthcare and that the ERS guideline incorporates this within their recommendations to be published in 2019. 
Due to the high number of active patients keen to share their views to improve treatment, we also encourage the sarcoidosis health and research community to partner with sarcoidosis patient organisations and to utilise this immense source of sarcoidosis lived experience to develop effective methods of measuring and incorporating QoL and functionality outcomes as a way of improving sarcoidosis outcomes and future treatment.

Robert P. Baughman ${ }^{1}$, Rita Barriuso ${ }^{2}$, Kelli Beyer ${ }^{3}$, Jeanette Boyd ${ }^{4}$, Johann Hochreiter ${ }^{5}$, Chris Knoet ${ }^{6}$, Filippo Martone ${ }^{7}$, Bernd Quadder ${ }^{8}$, Jack Richardson ${ }^{9}$, Ginger Spitzer ${ }^{3}$, Dominique Valeyre ${ }^{10}$ and Gianluca Ziosi ${ }^{7}$

${ }^{1}$ University of Cincinnati Medical Center, Cincinnati, OH, USA. ${ }^{2}$ Asociación Nacional de Enfermos de Sarcoidosis, Madrid, Spain. ${ }^{3}$ Foundation for Sarcoidosis Research, Chicago, IL, USA. ${ }^{4}$ European Lung Foundation, Sheffield, UK. ${ }^{5}$ Lungenfibrose Forum Austria, Vienna, Austria. ${ }^{6}$ Sarcoïdose Belangenvereniging Nederland (SBN), Valkenburg, The Netherlands. ${ }^{7}$ Amici Contro la Sarcoidosi Italia, Bologna, Italy. ${ }^{8}$ Deutsche Sarkoidose-Vereinigung e.V. (DSV), Health Policy and Sarcoidosis Research, Meerbusch, Germany. ${ }^{9}$ SarcoidosisUK, London, UK. ${ }^{10}$ Hopital Avicenne Service de Pneumologie, Bobigny, France.

Correspondence: Robert P. Baughman, University of Cincinnati Medical Center, Cincinnati, OH, USA. E-mail: bob.baughman@uc.edu

Received: Aug 282018 | Accepted: Nov 062018

Conflict of interest: R.P. Baughman reports grants from Gilead (grant to study sarcoidosis associated pulmonary hypertension), Bayer (grant to study sarcoidosis associated pulmonary hypertension), National Institutes of Health (grant to study sarcoidosis therapy), Foundation for Sarcoidosis Research (grant to study sarcoidosis) and Genentech (grant to study sarcoidosis associated fibrosis), and grants and personal fees from Mallinckrodt (grant support to study sarcoidosis, consultant and speaker fees), outside the submitted work. R. Barriuso has nothing to disclose. K. Beyer has nothing to disclose. J. Boyd is an employee of the European Lung Foundation. J. Hochreiter has nothing to disclose. C. Knoet has nothing to disclose. F. Martone has nothing to disclose. B. Quadder has nothing to disclose. J. Richardson is an Employee of SarcoidosisUK - representing sarcoidosis patients in the UK. G. Spitzer has nothing to disclose. D. Valeyre reports personal fees from Boehringer Ingelheim (member of scientific advisory boards on IPF), Roche (member of scientific advisory boards on IPF), AstraZeneca (presentation in Avancées de pneumologie), and BI and Roche (support for accommodation and transportation at scientific meetings: ERS, ATS and CPLF), outside the submitted work. G. Ziosi has nothing to disclose.

\section{References}

1 European Medical Agency (EMA). Public summary of opinion on orphan designation. EMA/COMP/554715/2013. www.ema.europa.eu/documents/orphan-designation/eu/3/13/1191-public-summary-opinion-orphan-designation-1pyr-1-glu-l-gln-l-leu-l-glu-1-arg-1-ala-1-leu-1-asn_en.pdf Date last updated: October 29, 2013.

2 Denning DW, Pleuvry A, Cole DC. Global burden of chronic pulmonary aspergillosis complicating sarcoidosis. Eur Respir J 2013; 41: 621-626.

3 Drent M, Costabel U, eds. Sarcoidosis (ERS Monograph). Sheffield, European Respiratory Society, 2005.

4 Hendriks C, Drent M, De Kleijn W, et al. Everyday cognitive failure and depressive symptoms predict fatigue in sarcoidosis: A prospective follow-up study. Respir Med 2018; 138S: S24-S30.

5 Drent M, Stookappe B, Hoitsma E. Consequences of Sarcoidosis. Clin Chest Med 2015; 36: 727-737.

6 Moor CC, van Manen MJG, van Hagen PM, et al. Needs, perceptions and education in sarcoidosis: a live interactive survey of patients and partners. Lung 2018; 196: 569-575.

7 European Lung Foundation/European Respiratory Society. Online survey: Sarcoidosis treatment and outcomes: what is most important to you? 2018. www.surveymonkey.com/r/Preview/?sm=Xx2rw_2BFgeFmjWLPJHyKFSL_ 2FGkgBGq0Etg8oJa8_2Fgx_2BhrNQw8VqA5UIgMkFwqvqD_2F

8 Baughman RP, Judson MA, Wells A. The indications for the treatment of sarcoidosis: Wells law. Sarcoidosis Vasc Diffuse Lung Dis 2017; 34: 280-282.

9 Maier LA, Crouser ED, Martin WJ, et al. Executive summary of the NHLBI workshop report: leveraging current scientific advancements to understand sarcoidosis variability and improve outcomes. Ann Am Thorac Soc 2017; 14: Suppl. 6, S415-S420. 